\title{
Vascularização arterial da base do encéfalo do gerbil (Meriones unguiculatus Milne-Edwards, 1867)*
}

\section{Arterial vascularization of the base of the cerebrum of mongolian gerbils (Meriones unguiculatus Milne-Edwards, 1867)}

\author{
Herson da Silva Costa, ${ }^{* *}$ Gleidson Benevides de Oliveira, ${ }^{* * *}$ Radan Elvis Matias de Oliveira, ${ }^{* *}$ Hélio Noberto de Araújo Júnior, ${ }^{* * * *}$ \\ Zuliete Aliona Araújo de Souza, ${ }^{* * * * *}$ Carlos Eduardo Bezerra de Moura, ${ }^{* * * * *}$ Moacir Franco de Oliveira ${ }^{* * * * *}$
}

\section{Resumo}

Objetivou-se descrever e sistematizar as artérias da base do encéfalo. Foram utilizados dez gerbils que se encontravam armazenados em freezer no laboratório de Morfofisiologia Animal Aplicada da Universidade Federal Rural do Semiárido. Os animais foram descongelados, incisados na linha mediana para identificação do ventrículo esquerdo, o qual foi perfurado e injetados $3 \mathrm{ml}$ de Látex Neoprene 650 na concentração de $20 \%$ e no pigmento vermelho. Posteriormente aguardou-se cerca de 1 minuto em virtude da polimerização do Látex e logo após foram fixados por imersão em solução aquosa de Formaldeído a 3,7\% e após 72 horas, tiveram os encéfalos removidos da calota craniana para análise de sua superfície ventral. Foi visto que o sistema vertebrobasilar e carótico estiveram presentes em todos os animais analisados, mostrando-se responsável pela vascularização do encéfalo caudal e rostral, respectivamente. As principais artérias observadas no modelo padrão incluem as ímpares: basilar, espinhal ventral, ramo medial da artéria cerebral rostral e as pares: vertebral, cerebelar caudal, cerebelar média, trigeminal, cerebelar rostral, ramo terminal da basilar, carótida interna, cerebral caudal, hipofisária, cerebral média, cerebral rostral, lateral do bulbo olfatório e etmoidal interna. Conclui-se com base nos resultados que a irrigação da superfície ventral encefálica do gerbil foi suprida pelos sistemas vertebro-basilar e carótico de forma equivalente, pertencendo assim ao modelo do tipo II. O circuito arterioso apresentou-se aberto caudalmente e fechado rostralmente, fato que difere do relatado em roedores como capivara, cutia, preá, chinchila e nutria.

Palavras-chave: artérias cerebrais, circuito arterioso, roedor, sistema carótico, sistema vertebrobasilar.

\begin{abstract}
The objective was to describe and systematize the arteries of the base of the brain. Ten gerbils were used, which were stored in a freezer in the Laboratory of Applied Animal Morphophysiology of the Federal Rural Semi-Arid University. The animals were thawed, incised in the midline to identify the left ventricle, which was punctured and injected $3 \mathrm{ml}$ of Latex Neoprene 650 at a concentration of $20 \%$ and red pigment. After about one minute the polymerization of the Latex was delayed and afterwards fixed by immersion in $3.7 \%$ aqueous Formaldehyde solution and after 72 hours the brain was removed from the skull cap for analysis of its ventral surface. It was observed that the vertebro-basilar and carotid system were present in all animals analyzed, being responsible for the vascularization of the caudal and rostral encephalon, respectively. The main arteries observed in the standard model include the odd: basilar, ventral spinal, medial branch of the rostral cerebral artery and, the pairs: vertebral, cerebellar caudal, cerebellar medial, trigeminal, cerebellar rostral, terminal branch of the basilar, internal carotid, caudal cerebral, Pituitary, middle cerebral, rostral, lateral, olfactory and internal ethmoidal. Based on the results, the irrigation of the ventral brain surface of the gerbil was supplied by the vertebro-basilar and carotic systems in an equivalent way, thus belonging to the type II model. The arterial circuit was open caudally and closed rostrally, a fact that differs from that reported in rodents such as capybara, agouti, cavy, chinchilla and nutria.
\end{abstract}

Keywords: arterial circuit, cerebral arteries, carotid system, rodent, vertebro-basilar system.

*Recebido em 17 de setembro de 2015 e aceito em 28 de março de 2017.

**Mestrando em Ciência Animal pelo Programa de Pós-graduação em Ciência Animal, Universidade Federal Rural do Semi-Árido - UFERSA; Mossoró/ RN/Brasil.

***Doutor em Ciência Animal; Mossoró/RN/Brasil.

****Discente do curso em Medicina Veterinária; UFERSA; Centro de Ciências Biológicas e da saúde; Mossoró/RN/Brasil.

*****Professora, Doutora em Ciência Animal; Faculdade de Enfermagem e de Medicina Nova Esperança - FACENE; Mossoró/RN/Brasil

****** Professor, Doutor do Curso em Medicina Veterinária; UFERSA; Centro de Ciências Biológicas e da saúde; Mossoró/RN/Brasil.

Autor para correspondência: herson-costa@hotmail.com 


\section{Introdução}

O gerbil é um roedor pertencente à família Muridae, encontrado no interior da Mongólia, sul da Sibéria, norte da China e atualmente podendo ser encontrado por todo o mundo. Caracteriza-se por apresentar um corpo alongado de $120 \mathrm{~mm}$ de comprimento em média, cauda longa, que pode atingir $120 \mathrm{~mm}$ de comprimento. Possuem orelhas curtas, pelagem que varia do branco ao rosa, ou amarelo com raios de cinza e hábito diurno, alimentando-se de grãos, gramíneas, frutas e legumes (AGREN et al., 1989). Esta espécie foi levada inicialmente da Mongólia para os Estados Unidos em 1954, com o intuito de ser utilizado como modelo experimental. Isso resultou, ao longo dos anos, na criação de uma variedade de cores e padrões para esses animais (SCHWENTKER, 1963). No entanto, devido às características sociáveis de serem divertidos e brincalhões, fáceis de cuidar, conviver bem com outros roedores e ter boa aparência, a espécie tornou-se de estimação, deixando de ser usado em pesquisas científicas, fato que resultou na carência de dados sobre sua morfologia, em especial sobre a irrigação arterial do sistema nervoso.

Estudos a cerca da vascularização encefálica e circuito arterioso, já foram descritos em espécies domésticos como equino (SOUZA et al., 2010), suíno (LIMA et al., 2005; MATOS et al., 2013), cão (ALCÂNTARA; PRADA, 1996a; ALCÂNTARA; PRADA, 1996b), gato (LIMA et al., 2006; LIMA et al., 2010; GOMES et al., 2015) e coelho (SOUZA; CAMPOS, 2013). Em espécies silvestres citam-se dados de estudos em irara (MACEDO et al., 2014), macaco-prego (FERREIRA; PRADA, 2009), quati (BARREIRO et al., 2012) e tamanduá-mirim (LIMA et al., 2013). Já em roedores silvestres, referenciam-se estudos coman capivara (RECKZIEGEL et al., 2001), chinchila (ARAUJO; CAMPOS, 2005), porco-espinho (AYDIN et al., 2005), nutria (AZAMBUJA, 2007), esquilo vermelho (AYDIN, 2008), toupeira-rato (AYDIN et al., 2008), cutia (SILVA et al., 2016) e preá (COSTA et al., 2014), sendo que dentre esses animais o tipo de vascularização encefálica e circuito arterioso apresentam distinções.

No gerbil, devido à sua utilização por muito tempo como "animal pet", acarretou-se assim, um déficit sobre sua morfologia. Objetivou-se então descrever e sistematizar as artérias da base do encéfalo, visando estabelecer um modelo padrão, promover o sucesso em intervenções cirúrgicas de interesse experimental no encéfalo, contribuir para a biologia da espécie e fornecer dados para a anatomia comparada.

\section{Material e métodos}

Foram utilizados dez animais (5 fêmeas e 5 machos), adultos (três meses de idade) e pesando de 150 a 200 gramas, provenientes de outras pesquisas (Parecer da Comissão de Ética no Uso de Animais - CEUA $n^{\circ}$ 04/2014, processo $n^{\circ}$ 23091.005376/2013-67) que foram doados ao laboratório de Morfofisiologia Animal Aplicada da Universidade Federal Rural do Semiárido e encontravam-se armazenados em freezer. Os animais foram descongelados e em seguida realizada uma incisão longitudinal mediana na região torácica para identificação do sistema cardiovascular. Depois, injetaram-se $3 \mathrm{ml}$ de Látex Neoprene 650 (Du Pont do Brasil S.A.) a 20\% com pigmento vermelho (Globo S.A. Tintas e Pigmentos) diretamente no ventrículo esquerdo com auxílio de seringa acoplada a agulha 25x7 Bico Slip. Neste momento, o local foi pressionado com pinça crile para evitar o retorno e extravasamento do Látex; em seguida, procedeu-se à retirada da seringa acoplada à agulha e aguardou-se cerca de 1 minuto para que ocorresse a polimerização do Látex. Posteriormente os animais foram fixados por imersão em solução aquosa de Formaldeído a 3,7\% e após 72 horas, iniciaram-se as dissecações, seguido da remoção dos encéfalos da calota craniana para análise de sua superfície ventral, usando quando necessário as identificadas, fotografadas, esquematizadas e nominadas segundo nomenclatura adotada pelo International Committee on Veterinary Gross Anatomical Nomenclature (2012). Os resultados foram expressos com base em anatomia descritiva e avaliados com base no cálculo de porcentagem simples.

\section{Resultados e discussão}

Os estudos acerca da vascularização arterial da base do encéfalo são antigos e aqueles realizados por De Vriese no ano de 1905, são pioneiros e de grande relevância científica, pois retratam modelos preconizados e muito utilizados nos dias atuais. Assim, De Vriese (1905), em seu estudo, classificou a irrigação da base do encéfalo dos mamíferos como sendo do tipo I, se a irrigação é exclusivamente via sistema carótico, do tipo II, se a irrigação ocorrer pelo sistema carótico e sistema vertebrobasilar, com distribuição igual entre os dois sistemas ou a predominância de um sobre o outro e do tipo III, se a irrigação do encéfalo ocorrer exclusivamente via sistema vertebrobasilar (Tabela 1).

De acordo com os resultados obtidos neste estudo, constatou-se que a vascularização encefálica do gerbil se dar pelo sistema corótico e também pelo sistema vertebrobasilar em todos os animais, o que os classificam como animais de irrigação encefálica do tipo II. Estes sistemas emitem vasos que se distribuem nas regiões de medula espinhal, medula oblonga, corpo trapezoide, ponte, pedúnculo cerebral, cerebelo, lobo piriforme, corpo mamilar, túber cinério, quiasma óptico, nervo óptico, trígono olfatório e bulbo olfatório (Figura 1).

Assim, no que se refere ao tipo vascular identificado no gerbil, este foi semelhante ao descrito em roedores como cutia (SILVA et al., 2016) e toupeira rato (AYDIN et al., 2008), pois estes animais apresentam a base do encéfalo irrigada por um sistema constituído pela artéria carótida interna e sistema vertebrobasilar. Iguala-se também àquele descrito em carnívoros como o cão e o gato segundo descrevem (ALCÂNTARA; PRADA, 1996a; ALCÂNTARA; PRADA, 1996b) e (LIMA et al., 2006; LIMA et al., 2010), respectivamente. Porém, distam do que descreve Aydin et al. (2005) e Azambuja (2007) ao tratarem sobre a vascularização do encéfalo em porco-espinho e nutria, respectivamente, visto que, nestes roedores, a irrigação da base do encéfalo, faz-se de forma independente exclusivamente pelo sistema vertebrobasilar, o que os define como sendo animais com irrigação encefálica do tipo III.

Neste contexto, vale ressaltar que a vascularização da base do encéfalo do gerbil, foi composta pelas artérias ímpares: basilar, espinhal ventral, ramo medial da artéria cerebral rostral e as pares: vertebral, cerebelar caudal, cerebelar média, trigeminal, cerebelar rostral, ramo terminal da basilar, carótida interna, cerebral caudal, hipofisária, cerebral média, cerebral rostral, lateral do bulbo olfatório e etmoidal interna. Dessa forma, logo caracterizou três diferentes modelos de distribuição vascular em gerbils (Figura 2). 
Tabela 1: Vascularização arterial da base do encéfalo em roedores, com base na classificação de De Vriese (1905)

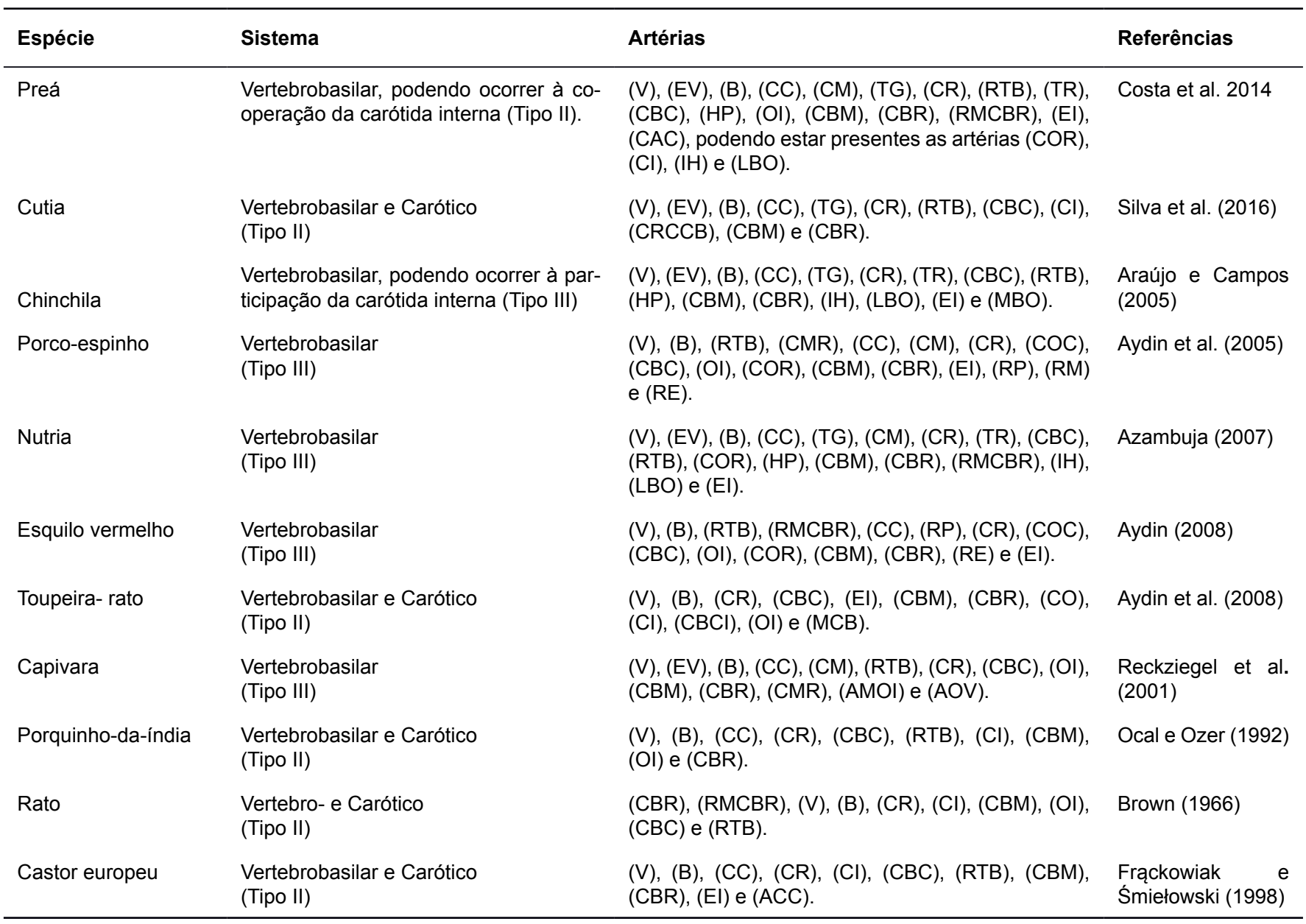

Vertebrais $(\mathrm{V})$, espinhal ventral (EV), artérias basilar (B), carótida interna $(\mathrm{Cl})$, cerebelar caudal $(\mathrm{CC})$, cerebelar média (CM), cerebelar rostral (CR), artérias mesencefálicas (AM), cerebral caudal (CBC), cerebral caudal interna ( $\mathrm{CBCl}$ ), cerebral média (CBM), cerebral rostral (CBR), corióidea rostral (COR), corióidea caudal (COC), corioidea (CO), etmoidais internas (EI), hipofisárias (HP), inter-hemisférica rostral mediana (IH), mediana cerebral (MCB), oftálmicas interna (OI), laterais do bulbo olfatório (LBO), mediais do bulbo olfativo (MBO), arterias para o corpo caloso (ACC), ramos terminais da basilar (RTB), ramo medial da cerebral rostral (RMCBR), tectal rostral (TR), trigeminais (TG), ramos para ponte (RP), ramos para mesencéfalo (RM), ramos estriados (RE), ramos corticais $(R C)$, anastomose entre maxilar e oftálmicas internas (AMOI), anastomose entre occipital e vertebrais (AOV), anastomoses das redes admiráveis epidurais caudais (AAC), ramos caudais da carótida do encéfalo (RCCE) e o circuito arterioso cerebral (CAC) distribuídos pelos antímeros Direito (D) e esquerdo (E).

No que tange ao circuito arterioso cerebral do gerbil, este se apresentou aberto caudalmente e fechado rostralmente em todos os animais analisados. Os referidos achados são diferentes, por exemplo, do descrito por Silva et al. (2016) na cutia, o qual descreve que nestes animais, o circuito arterioso da base do encéfalo é completamente fechado e, ainda do relatado em preás (COSTA et al., 2014) e irara (MACEDO et al., 2014), mamíferos em que os autores observaram que o circuito arterioso é completo e fechado (caudalmente e rostralmente). Diferem ainda do presente estudo, os achados para o nutria (AZAMBUJA, 2007) e chinchila (ARAÚJO; CAMPOS, 2005), já que nestes animais o circuito arterioso é aberto rostralmente. Fizeram parte do circuito arterioso do gerbil, os ramos terminais da basilar, carótidas internas, cerebrais caudais, cerebrais médias e rostrais e o ramo medial das artérias cerebrais rostrais, salientando-se a presença de interrupção vascular na região caudal do encéfalo (Figura 2).
No gerbil, a artéria espinhal ventral originou-se da artéria vertebral direita em todos os animais estudados e se projetava rostro-caudalmente na fissura mediana ventral da medula espinhal, sendo responsável por irrigar esta região (Figura 2). Em contrapartida Souza e Campos (2013) citam em estudos com artérias da base do encéfalo de coelhos, que a artéria espinhal ventral surge mais frequentemente da anastomose entre as artérias vertebrais direita e esquerda, podendo originar-se somente da artéria vertebral esquerda ou direita.

Quanto à artéria vertebral direita e esquerda, em gerbils, estas sofriam anastomose e originava a artéria basilar que seguia um trajeto caudorrostral, ao longo da linha mediana ventral do rombencéfalo, sendo um vaso retilíneo em (90\%) dos animais e sinuoso em $10 \%$ dos espécimes (Figura 2). Semelhante ao presente estudo foi evidenciado no nutria (AZAMBUJA, 2007). Já em preás (COSTA et al., 2014), constatou-se somente a presença deste vaso, na forma retilínea. 


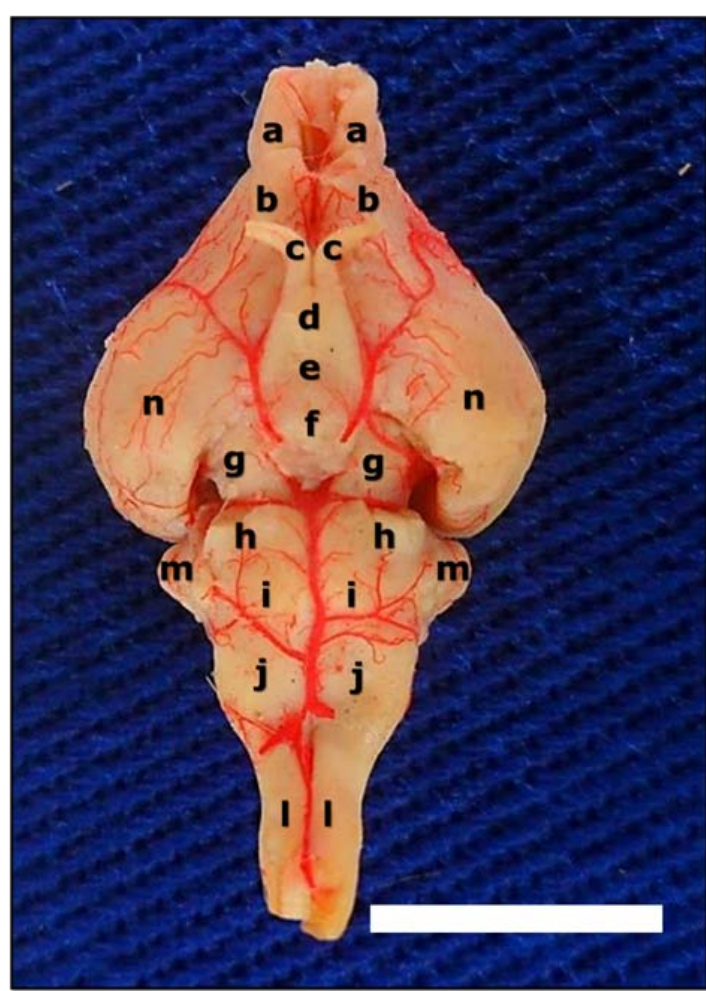

Figura 1: Estruturas da base do encéfalo do gerbil, constatando-se o bulbo olfatório (a), trígono olfatório (b), nervo óptico (c), quiasma óptico (d), túber cinério (e), corpo mamilar (f), pedúnculo cerebral $(\mathrm{g})$, ponte $(\mathrm{h})$, corpo trapezoide (i), medula oblonga $(j)$, medula espinhal $(\mathrm{I})$, cerebelo $(\mathrm{m})$ e lobo piriforme $(n)$. Barra: $1 \mathrm{~cm}$.
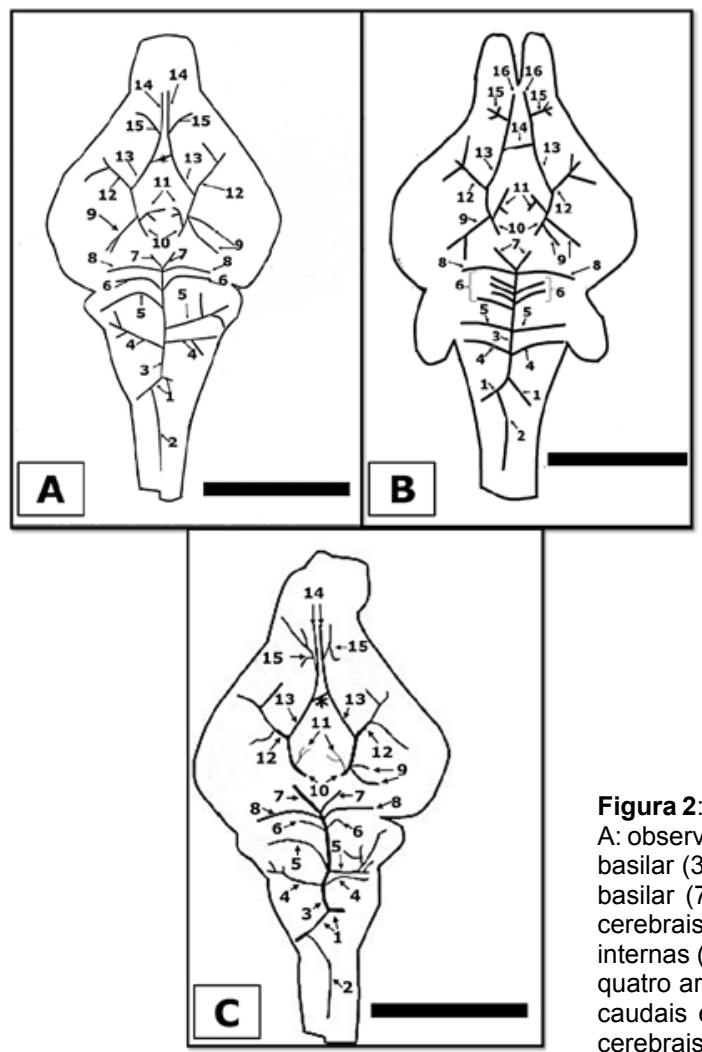

A partir da artéria basilar, emitiram-se colateralmente as artérias cerebelar caudal, cerebelar média, trigeminal e cerebelar rostral, em ambos os antímeros. Após atingir a extremidade rostral da ponte, a artéria basilar dividiu-se em dois ramos terminais, um direito e outro esquerdo, que se direcionavam laterorrostralmente (Figura 2).

Em relação às artérias cerebelares caudais (direita e esquerda) no gerbil, esta surgiu como um vaso único dorsalmente à medula oblonga, sendo responsável pela irrigação da porção caudal do cerebelo (Figura 2). Esta descrição também foi feita por Silva et al. (2016) ao estudar a base do encéfalo de cutias. Comportamento diferente foi observado por Araújo e Campos (2005), em pesquisas com chinchilas, os quais citam que este vaso, logo após ser emitido pela artéria basilar, projeta-se lateralmente para o interior da fissura transversa, distribuindo-se no mesencéfalo e no polo caudal dos hemisférios cerebrais.

Referindo-se às artérias trigeminais, no gerbil foi observada como vaso único na maioria $(90 \%)$ dos animais estudados, onde seguiu na extremidade rostral da ponte e projetava-se lateralmente, alcançando e irrigando a base aparente do nervo trigêmeo, em ambos os antímeros, porém destacase a presença de uma variação, já que, a artéria trigeminal direita foi emitida como vaso quádruplo, enquanto a trigeminal esquerda foi emitida como vaso duplo, situação observada em um animal (10\%) (Figura 2). Diferentemente do presente estudo, foi inferido para o nutria (AZAMBUJA, 2007) e o coelho (SOUZA; CAMPOS, 2013), os quais evidenciaram este vaso como sendo único.

No que concerne à participação da artéria carótida interna na irrigação encefálica no gerbil, esta esteve presente em todos os animais, em ambos os antímeros, surgindo na altura do corpo mamilar, o qual emitiu as artérias hipofisárias e cerebrais caudal, média e rostral (Figura 2). Tais achados distam do que descreve Araújo e Campos (2005) na chinchila, onde constataram a presença da artéria carótida interna apenas em dois animais de um total de 31 espécimes analisados, surgindo no terço médio da medula oblonga. Diferente ainda foi observado por Azambuja (2007) em trabalhos com nutria, o qual descreve a ausência desse vaso como artéria na base do encéfalo.

No que se refere à artéria cerebral caudal do gerbil, esta surgiu a partir das artérias carótidas interna e seguiu laterodorsalmente ao pedúnculo cerebral, distribuindo-se no tálamo, estria medular, glândula pineal e no hemisfério cerebral. Comumente, a cerebral caudal direita e esquerda correspondia a um único vaso, que se bifurcava após sua emissão ou ao longo do seu caminho, enquanto em um animal, a cerebral caudal esquerda surgia de forma dupla, já em outra amostra, observou-se a ausência da artéria cerebral caudal direita (Figura 2). Estes achados corroboram com os estudos de Araújo e Campos (2005) na chinchila, quando relatam que esta artéria pode apresentar-se como vaso duplo. Já em coelhos (SOUZA; CAMPOS, 2013) e em gatos (LIMA et al., 2010), estes autores observaram que a artéria cerebral caudal teve origem a partir da anastomose entre as artérias carótida interna e o ramo terminal da artéria basilar, diferente do verificado no gerbil, onde não se verificou comunicações entre os ramos terminais da artéria basilar e carótidas.

Figura 2: Desenhos esquemáticos dos modelos de distribuição vascular da base do encéfalo em gerbils. A: observa-se o modelo vascular padrão, onde se constata as artérias vertebrais (1), espinhal ventral (2), basilar (3), cerebelares caudais (4), cerebelares médias (5), trigeminais (6), ramos terminais da artéria basilar (7), cerebelares rostrais (8), cerebrais caudais (9), carótidas internas (10), hipofisárias (11), cerebrais médias (12), cerebrais rostrais (13), ramo medial das artérias cerebrais rostrais ${ }^{*}$ ), etmoidais internas (14) e laterais do bulbo olfatório (15). B: observa-se uma variação anatômica pela presença de quatro artérias trigeminais direita e duas esquerdas (6) e, origem separada de duas artérias cerebrais caudais esquerdas. C: evidencia-se outra variação anatômica onde se observa apenas as artérias cerebrais caudais esquerdas (9) e artéria basilar sinuosa (3). Barra: $1 \mathrm{~cm}$. 
No presente estudo, observou-se que a artéria cerebral média direita e esquerda, teve origem a partir das carótidas internas em $100 \%$ das amostras, da mesma forma, Lima et al. (2013) relatam no tamanduá-mirim que esta artéria surge da carótida interna. Já Silva et al. (2016) estudando cutias, citam a origem da artéria cerebral média direita e esquerda, a partir do ramo comunicante rostral da carótida cerebral, na altura do trato óptico, diferindo assim dos nossos achados.

Em gerbils, a artéria cerebral rostral (direita e esquerda) mostrouse como sendo o ramo terminal das carótidas internas, surgindo da bifurcação que originou a artéria cerebral média, o qual foi responsável pela emissão do ramo medial da artéria cerebral rostral e posteriormente, as artérias laterais do bulbo olfatório e etmoidais internas (Figura 2). Diferentemente do encontrado no gerbil, foi relatado no nutria (AZAMBUJA, 2007), por ser citado que a cerebral rostral se origina dos ramos terminais da basilar. Já De Vriese (1905) estudando os mamíferos, afirmou que dados relativos à ontogênese e filogênese indicam que a

\section{Referências}

AGREN, G.; ZHOU, P.; ZHONG, W. Ecology and social behaviour of Mongolian gerbils, Meriones unguiculatus, at Xilinhot, Inner Mongolia, China. Animal Behavior, v. 37, p.11-27, 1989. Disponível em: < http://www.sciencedirect.com.sci-hub.org/ science/article/pii/000334728990002X>. Acesso em: 16 set. 2015.

ALCÂNTARA, M. A.; I. L. S. PRADA. Arteries of the basis of encephalon in dogs (canis familiares, Linnaeus, 1758). I. Anatomical study of sources and behavior. Brazilian journal of veterinary Research and Animal Science, v. 33, n. 2, p. 67-71, 1996 a. Disponível em: < http://www.revistas.usp.br/bjvras/article/ view/50160/54275>. Acesso em: 15 nov. 2016.

ALCÂNTARA, M. A.; PRADA, I. L. S. Arteries of the basis of the encephalon in dogs (Canis familiaris, Linnaeus, 1758). II. Formation and behaviour of the encephalon arterial circuit. Brazilian journal of veterinary Research and Animal Science, v. 33, n. 2, p. 72-76, 1996 b. Disponível em: < http://www.revistas. usp.br/bjvras/article/view/50161/54276>. Acesso em: 15 nov. 2016.

ARAÚJO, A.C.P.; CAMPOS, R. A systematic study of the brain base arteries and their blood supply sources in the chinchilla (Chinchilla lanigera - Molina 1782). Braz. J. morphol. Sci., v. 22, n. 4, p. 221-232, 2005. Disponível em: <http://coral.ufsm.br/ ccrrevista/normas.htm>. Acesso em: 03 set. 2015.

AYDIN, A.; YILMAZ, S.; OZKAN, Z. E.; ILGUN, R. Morphological Investigations on the Circulus Arteriosus Cerebri in Mole-Rats (Spalax leucodon). Anat. Histol. Embryol., v.37, p.219- 222, 2008. Disponível em:< http://onlinelibrary.wiley.com.sci-hub.org/ doi/10.1111/j.1439-0264.2007.00834.x/full>. Acesso em: 03 set. 2015.

AYDIN, A.; YILMAZ, S.; DINC, G.; OZDEMIR, D.; KARAN, M. The morphology of circulus arteriosus cerebri in the porcupine (Hystrix cristata). Veterinary Medicine Czech., v.50, p.131-135, 2005.

Disponível em:< http://agriculturejournals.cz/publicFiles/61272. pdf $>$. Acesso em: 03 set. 2015.

AYDIN, A. The morphology of circulus arteriosus cerebri in the red squirrel (Sciurus vulgaris. Veterinarni Medicina, v. 53, n. 5, p.272276, 2008. Disponível em:< http://81.0.228.28/publicFiles/02251. pdf>. Acesso em: 03 set. 2015. artéria cerebral rostral é o único ramo terminal da artéria carótida interna, semelhante ao presente estudo. Araújo e Campos (2005), na chinchila e Silva et al. (2016) na cutia afirmam que a artéria cerebral rostral pode estar ausente, situação não observada no gerbil.

\section{Conclusões}

A base do encéfalo do gerbil é suprida pelos sistemas vertebrobasilar e carótico de forma equivalente e, assim classificado como tipo II de irrigação encefálica, constatação que diverge de resultados descritos para outros roedores, como o nutria, capivara, chinchila, esquilo vermelho e porco-espinho e estando mais próximo dos roedores como preás, toupeirarato, cutia, ratos, porquinho-da-índia e castor europeu. Já o circuito arterioso da espécie apresentou-se aberto caudalmente e fechado rostralmente, em todos os animais, contornando lateralmente o corpo mamilar, o túber cinério e o quiasma óptico.

AZAMBUJA, R.C. de. Sistematização das artérias da base do encéfalo e suas fontes de suprimento sangüíneo em nutria (Myocastor coypus). Acta Scientiae Veterinariae. (UFRGS. Impresso), v. 35, n. 2, p. 277-278, 2007. Disponível em:< http:// www.ufrgs.br/actavet/35-2/resumo11.pdf>. Acesso em: 15 nov. 2016.

BARREIRO, J.R.; CARVALHO, A.F.; FRANCIOLLI, A.L.R.; FERREIRA, G.J.B.C.; FERREIRA, J.R.; AMBRÓSIO, C.E.; MIGLINO, M.A. Morfologia dos vasos da base do encéfalo do quati (Nasua nasua). Pesq. Vet. Bras. v. 32, n. 6, p. 567-572, 2012. Disponível em:< http://www.scielo.br/pdf/pvb/v32n6/ v32n6a16.pdf>. Acesso em: 04 set. 2015.

BROWN, J.O. The morphology of circulus arteriosus cerebri in rats. The Anatomical Record, v. 156, n. 1, p. 99-106, 1966. Disponível em:< http://onlinelibrary.wiley.com.sci-hub.org/ doi/10.1002/ar.1091560112/abstract>. Acesso em: 16 set. 2015.

COSTA, H.S.; OLIVEIRA, R.E.M.; CÂMARA, F.V.; BEZERRA, F.V.F.; OLIVEIRA, G.B.; ARAÚJO JÚNIOR, H.N.; SILVA, A.R.; ALBUQUERQUE, J.F.G.; OLIVEIRA, M.F. Vascularização arterial da base do encéfalo de preá (Galea spixii Wagler, 1831). In: XX SEMINÁRIO DE INICIAÇÃO CIENTÍFICA DA UFERSA - SEMIC, 2014. Anais do XX Seminário de Iniciação Científica da UFERSA - SEMIC, 2014.

DE VRIESE, B. Sur la signification morphologique des artères cérébrales. Archives de Biologie, Liège, v. 21, p. 357-457, 1905.

FERREIRA, J.R.; PRADA, I.L.S. O sistema carótico do encéfalo do Cebus apella sp., Linnaeus, 1766. Biota Neotropica.

Campinas, v. 9, n. 1, p. 285-292, 2009. Disponível em:< http:// www.scielo.br/pdf/bn/v9n1/32.pdf>. Acesso em: 04 set. 2015.

FRACKOWIAK, H.; ŚMIEŁOWSKI, J. Cephalic arteries in the European beaver Castor fiber, Acta Theriologica v. 43, n. 2, p. 219-224, 1998. Disponível em:< http://rcin.org.pl/Content/12766/ BI002_27002_Cz-40-2_Acta-T43-nr19-219-224_o.pdf>. Acesso em: 04 set. 2015 .

GOMES, M.S.; MORAES, S.O.S.; FIGUEIREDO, M.A. Craniometria, morfometria do encéfalo e tipologia do circuito arterial da base do encéfalo em gatos. Revista Brasileira de Ciência Veterinária (Impresso), v. 22, n.1, p. 3-9, 2015. Disponível em:< http://www.uff.br/rbcv/ojs/index.php/rbcv/article/view/718>. Acesso em: 15 nov. 2016. 
INTERNATIONAL COMMITTEE ON VETERINARY GROSS ANATOMICAL NOMENCLATURE. Nomina Anatomica Veterinária. 5.ed. Knoxville: World Association on Veterinary Anatomist, 2012. $177 \mathrm{p}$.

LIMA, E.M.M.; SEVERINO, R.S.; SILVA, F.O.C.; DRUMMOND, S.S.; BOMBONATO, P.P.; CAMPOS, D.B.; RODRIGUES, G.S. Artérias da base do encéfalo em suínos da linhagem camborough 22. Biosci. J., Uberlândia, v. 21, n. 2, p. 137-147, 2005. Disponível em:< http://www.seer.ufu.br/index.php/biosciencejournal/article/ view/6600/4333>. Acesso em: 16 set. 2015.

LIMA, E.M.M.; PRADA, I.L.S.; SILVA, F.O.C.; SEVERINO, R.S.; SANTOS, A.L.Q.; DRUMMOND, S.S.; RODRIGUES, G.S. Estudo anatômico das artérias da base do encéfalo em gatos. Ars Veterinária, Jaboticabal, v. 22, n.1, p.001-007, 2006. Disponível em:< http://repositorio.unb.br/bitstream/10482/7721/1/ARTIGO_ EstudoAnat\%C3\%B4micoArt\%C3\%A9rias.pdf >. Acesso em: $0 \overline{4}$ set. 2015.

LIMA, E.M.M.; PRADA, I.L.S.; SILVA, F.O.C; SEVERINO, R.S.; SANTOS, A.L.Q.; BORGES, B.O.; PAIM, T.P.; VIANNA, A.R.C.B. Sistematização da origem, da distribuição e dos territórios da artéria cerebral caudal na superfície do encéfalo em gatos. Ciência Rural, v.40, n.9, p.1961-1965, 2010. Disponível em:< http://www.scielo.br/pdf/cr/v40n9/a721cr2964.pdf >. Acesso em: 04 set. 2015.

LIMA, A.R.; PEREIRA, L.C.; BRANCO, E. Anatomia do circuito arterial do encéfalo em Tamanduá-mirim. Ciência Rural, v. 43, n. 2, p. 277-282, 2013. Disponível em:< http://www.scielo.br/pdf/cr/ v43n2/a4613cr6005.pdf >. Acesso em: 04 set. 2015.

MATOS, S.A.; PASSOS, N.C.; HERNANDEZ, J.M.F.; PALHANO, H.B.; ANTUNES, M.S.; SCHERER, P.O. Estudo morfológico da vascularização arterial da base do encéfalo de suínos (Sus scrofa domesticus, Linnaeus, 1758) mestiços. Revista Brasileira de Medicina Veterinária, v. , n. 4, p. 365-370, 2013. Disponível em:< http://www.rbmv.com.br/pdf_artigos/29-11-2013_20-38RBMV030. pdf>. Acesso em: 04 set. 2015.
MACEDO, B.C.; BRANCO, E.; PEREIRA, L.C.; LIMA, A.R. Estudo anatômico do circuito arterial do encéfalo em Eira barbara - relato de dois casos. Biotemas, v. 27, n. 2, p.177-183, 2014. Disponível em:< http://dx.doi.org/10.5007/2175-7925.2014v27n2p177 >. Acesso em: 04 set. 2015.

OCAL, M.K.; OZER, M. The circulus arteriosus cerebri in the guinea pig. Annals of Anatomy-Anatomischer Anzeiger, v. 174, n. 3, p. 259-260, 1992. Disponível em:< http://www.sciencedirect. com.sci-hub.org/science/article/pii/S0940960211803651 >. Acesso em: 16 set. 2015.

RECKZIEGEL, S.H.; LINDEMANN, T.; CAMPOS, R., A systematic study of the brain base arteries in capybara (hydrochoerus hydrochaeris), Braz. J. morphol. Sci. v. 18, n. 2, p. 103-110, 2001. Disponível em:< http://jms.org.br/PDF/v18n2a04.pdf>. Acesso em: 04 set. 2015.

SILVA, R.S.B.; OLIVEIRA, G.B.; OLIVEIRA JUNIOR, C.M.; BEZERRA, F.V.F.; CÂMARA, F.V.; OLIVEIRA, R.E.M.; OLIVEIRA, M.F. Arterial vascularization of the brain of the agouti (Dasyprocta aguti Linnaeus, 1766). Semina: Ciências Agrárias, Londrina, v. 37 , n. 2, p. 773-784, mar./abr. 2016. Disponível em:< http://www.uel. br/revistas/uel/index.php/semagrarias/article/view/20200/18596>. Acesso em: 15 nov. 2016.

SOUZA, F.; CAMPOS, R. A systematic study of the brain base arteries in the rabbit (Oryctolagus cuniculus). Pesquisa Veterinária Brasileira, v. 33, n. 6, p. 796-806, 2013. Disponível em:< http:// www.scielo.br/pdf/pvb/v33n6/18.pdf>. Acesso em: 04 set. 2015.

SOUZA, A.V.P.; SILVA, B.X.; ANTUNES, M.S.; HERNANDEZ, J.M.F.; SCHERER, P.O.; FIGUEIREDO, M.A. Frequência da artéria caroticobasilar em equinos mestiços: estudo anatômico destinado a pesquisa experimental e ao diagnóstico por imagem. Pesquisa Veterinária Brasileira, v. 30, n. 8, p. 685-688, 2010. Disponível em:< http://www.scielo.br/pdf/pvb/v30n8/13.pdf>. Acesso em: 04 set. 2015.

SCHWENTKER, V. The gerbil, a new laboratory animal. Illinois Veterinarian, v. 6, n. 4, p. 5-9, 1963. 